\title{
Computer measurement of social motivation
}

\author{
STEVEN P. McNEEL, SANDRA WEBSTER, and JOHN HAUSFELD \\ Southern Ilinois University, Carbondale, Illinois 62901
}

\begin{abstract}
This study examined a computer implementation of an algebraic model which assumes that a person's utility for a given social outcome is a simple linear function of the outcomes to self and to the other person in the interaction. The computer estimated the subject's social motivation orientation based solely on his choices in a series of decomposed games. The computer-measured motives closely corresponded to the verbally stated reasons subjects gave. A central advantage of the present method is that social motivation can be studied in a unified fashion, without artificial restriction to single substantive categories such as aggression, altruism, or cooperation/competition.
\end{abstract}

In the past decade or so, many social psychologists have focused on studies in areas such as helping behavior, altruism, bystander intervention, cooperation/competition, and aggression. However, in spite of the fact that these behaviors seem to fall naturally on a pro- to antisocial dimension, investigators in these areas have not approached their research from a unifjed viewpoint. Instead, they have used a multitude of research paradigms. While such methodological heterogeneity is understandable given the "social relevance" interests of many social psychologists, it does not necessarily lead to the natural accumulation of knowledge or to the articulation of a unified theoretical perspective (e.g., see Kuhn, 1962).

Recently, Griesinger and Livingston (1973) have developed an approach which allows measurement in the same experimental paradigm of behaviors varying on a pro- to antisocial dimension. Their approach originates in the social motivation viewpoint of McClintock (e.g., 1972; Messick \& McClintock, 1968). This viewpoint stresses that social interaction cannot be understood properly unless we first obtain adequate estimates of the values which people place on available interaction outcomes, i.e., estimates of people's social motives. Griesinger and Livingston's method is a computer implementation of an algebraic model (with a simple geometric representation) which assumes that a person's utility for a given social outcome is a linear function of outcomes to self and to the other in the interaction.

Details of the procedure are presented by Griesinger and Livingston (1973). However, a very brief and general overview will be helpful here. The procedure utilizes pairs of choice stimuli (see Figure 1), where choice of one alternative assigns a certain outcome to self and another outcome to the other person in the interaction. Such decomposed game alternatives can be represented as an ordered pair of outcomes $(x, y)$ in a two-dimen-

This research was supported by furids from a special research project awarded to the senior author by the Southern Illinois University Office of Research and Projects. Requests for reprints should be sent to Steven P. McNeel, Department of Psychology, Southern Illinois University, Carbondale, Illinois 62901. This paper is sponsored by R. R. Schmeck, who takes full editorial responsibility for its content. sional space characterizing person X's and person Y's outcomes. Preference of one choice alternative (outcome pair) over another implies that the former alternative is closer (in this two-dimensional outcome space) to the person's most preferred outcome pair. As the experiment proceeds, the computer bases its presentation of choice stimuli on the subject's previous choices, such that alternatives are presented to the subject which more and more closely approximate his (her) most preferred choice alternative. It should be noted that, in order for the procedure to make logical and empirical sense, the choice stimuli to be presented must be restricted to those falling on the circumference of some fixed radius circle, with the origin at $(0,0)$ in the outcome space.

When the choice stimuli are finally indistinguishable to the subject (i.e., identical), their common value is a measure of the subject's most preferred social outcome. Now, imagine a line drawn from the origin of the twodimensional outcome space to the point representing this most preferred social outcome. The angle this line makes with the axis representing the subject's own outcome is the measure of social motivation given by the model. Hence, social motivation can easily vary from pure altruism ( $=90 \mathrm{deg}$ or 1.571 radians) through pure individualism ( $=0 \mathrm{deg}$ or 0 radians) to pure hostility ( $=-90 \mathrm{deg}$ or -1.571 radians). Cooperation and competition occupy intermediate points at $45 \mathrm{deg}(.785$ radians) and $-45 \mathrm{deg}$ ( -.785 radians), respectively. (The reader can diagram possibilities for himself.) A final output of the computer procedure is the number of choice trials required for the computer to converge on the social motivation estimate.

The research reported by Griesinger and Livingston (1973) demonstrates that the measured motivational orientations of subjects given very specific instructions (individualistic, cooperative, and competitive) corre-

Choice A Choice B

\begin{tabular}{lll}
\hline Own Outcome & 7 & 9 \\
Other's Outcome & 0 & 5 \\
\hline
\end{tabular}

Figure 1. Example decomposed game. 
spond to expectations. These data represent one kind of support for the general adequacy of the geometric model as a measurement instrument. The present study sought to obtain another kind of support. In general, there should be a correspondence between the verbally stated motives of subjects and the geometric model's estimate of social motivation. If such phenomenological data did not correspond in general with measured social motivation, serious problems would be raised. ${ }^{1}$

\section{METHOD}

A Digital PDP-12 computer was used to implement the geometric model. Subjects viewed the choice alternatives on an oscilloscope in a different room than the computer and indicated their choices by pressing one of two switches beneath the oscilloscope.

A postexperimental questionnaire contained a determination of the subject's motivation (subjects checked the appropriate motive of eight motives described or wrote out a description of their motive if none of the eight was appropriate) and 7-point ratings of: (1) six semantic differential mood scales, (2) perceived temperature, (3) concern with outcomes presented in the feedback display, and (4) desire to finish the experiment as quickly as possible. Subjects also indicated their current grade point average and answered the two critical items from the Interpersonal Judgment Scale (Byrne, 1971).

Forty-four males (32 introductory psychology students and 12 introductory sociology volunteers) were run one at a time, though each subject was given the impression that he was genuinely interacting with another person. This was done by using fictitious names in sign-up folders, by reference to the other person by name, and by a brief rigged interaction between the experimenter and the other over the intercom during the instructional period. The subject was left alene to read the instructions for about $20 \mathrm{~min}$. The instructions introduced the choice situation and told subjects to make the choice they considered best. The experimenter went to the subject's room and answered any questions by paraphrasing the instructions. When the experimenter left, the choice trials began.

Consistent with the procedure of Griesinger and Livingston (1973), each subject saw trial-by-trial feedback of the results of his own choice on that trial. This serves to highlight the subject's own control over outcomes, while not giving information about the other person's choices that could be used for strategic purposes. Finally, subjects filled out a postexperimental questionnaire and were completely debriefed. ${ }^{2}$

\section{RESULTS}

\section{Social Motivation}

Subjects were divided according to the motives they checked (or wrote out) ${ }^{3}$ on the postexperimental questionnaire. The motivational orientation data obtained from the geometric model are shown in Table 1. For comparison purposes, Table 1 also presents the radian measure that would result if subjects were "purely" cooperative, individualistic, or competitive. It can be seen that the motivational orientations correspond closely to the verbally stated motives. Of the three main motivational orientations, only for competitively (verbal) oriented subjects is the measured motive significantly different from the "pure" orientation $[t(11)=2.27, p<.05]$. Further, the four groups of subjects differ significantly from each other $[F(3,40)=13.60, p<.001]$, and each of the three main motive groups (verbal) differs significantiy from the others at the .01 level. Note also that 7 of the 13 subjects in the all-others group verbalized either aggression or a combination of aggression and individualism, and their measured orientation was the most negative of any group (-.570).

\section{Trials to Convergence}

Table 1 also presents the trials-to-convergence data from the geometric model. The four groups do not differ significantly from each other $[F(3,40)=2.39, n . s]$. This is an important point, demonstrating that choice consistency is not related to specific motivational orientation. However, it is obvious without a statistical test that subjects in each group take much longer to converge than the theoretical minimum of nine trials (see Griesinger \& Livingston, 1973, p. 185). Further, comparison of Table 1 with Griesinger and Livingston's Figures 11 and 12 (pp. 185-186) shows that the means obtained here are in the same range as their means for cooperative subjects, but somewhat larger for individualistic and competitive subjects. Thus, there seems to be somewhat more inconsistency in the present study than in the Griesinger and Livingston study, while both studies show more inconsistency in choices than would be expected if subjects made choices only according to the choice rule that is assumed by the geometric model.

\section{Questionnaire}

None of the questionnaire measures were significantly related to verbally stated social motivation.

\section{DISCUSSION}

The results of this study support the notion expressed by Livingston (Note 1) that the computer implementation of the

Table 1

Motivational Orientation (Radians) and Trials to Convergence as a Function of Verbally Stated Social Motive

\begin{tabular}{lcccc}
\hline & Cooperative & Individualistic & Competitive & All Others \\
\hline Number of Subjects & 8 & 11 & 12 & 13 \\
"Pure" Motivational Orientation & .785 & .000 & -.785 & -.425 \\
Mean Motivational Orientation & .712 & .109 & .278 & -.277 \\
Variance & .125 & .055 & .221 \\
Mean Trials to Convergence & 15.75 & 19.18 & 23.17 & 1.42 \\
Variance & 22.79 & 41.16 & 91.32 \\
\hline
\end{tabular}


geometric model is a useful measurement procedure even though, as the trials to criterion data show, subjects do not choose consistently in the precise way assumed by the model (convergence requires more than nine trials). As a next step in developing a unified measurement approach to studying social motivation, it would seem helpful to examine several variables whose influence on social motivation is relatively well understood. For example, manipulation of an instigator and an inhibitor of aggression (e.g., insult and possibility of social censure) could be informative. Finally, more interesting combinations of social motivation cues could be studied. For example, how can we expect social motivation to vary as a function of a factorial combination of an instigator toward aggression and a cue toward helping such as dependency? While many other relevant methodologies restrict the range of the subject's responses (e.g., the typical "aggression machine" does not allow the subject a clear helping response), the present methodology makes available a full range of responses. The central advantage to the researcher is obvious: Social motivation can be studied in a unified fashion, without artificial restriction to a single substantive category such as aggression, altruism, or cooperation/competition.

\section{NOTES}

1. It is recognized that verbal statements may sometimes represent rationalizations of one's behavior rather than genuine behavioral goals.

2. To include a richness of environmental factors that previous research suggested might be related to a subject's goals, two variables were manipulated (11 subjects per cell): type of cumulative outcome feedback (joint vs. separate own and other's) and ambient temperature (normal $=74^{\circ} \mathrm{F}$; hot $=90^{\circ} \mathrm{F}$ ).
However, though there is evidence for manipulation effectiveness (at least for the heat variable), the computer measurements and questionnaire data showed no interesting and significant results. Hence, these manipulations will not be discussed further.

3. The second author read each motive statement that was written out and categorized it using, as necessary, any of the eight motive categories available to the subject. Uncertainties were resolved in consultation with the first author. This categorization was done without knowledge of the subject's computer measured motivation.

\section{REFERENCE NOTE}

1. Livingston, J. W., Jr. On the measurement of social motivation in decomposed games. Unpublished doctoral dissertation. University of California, Santa Barbara, 1971.

\section{REFERENCES}

Byrne, D. The attraction paradigm. New York: Academic Press. 1971.

Griesinger, D., \& Livingston, J. Toward a model of interpersonal motivation in experimental games. Behavioral Science, 1973, 18, 173-188.

Kunn. T. S. The structure of scientific revolutions. Chicago: University of Chicago Press, 1962.

MCClintock, C. Social motivation-A set of propositions. Behavioral Science, 1972, 17, 438-455.

Messick, D. \& \& McClintock, C. Motivational bases of choice in experimental games. Journal of Experimental Social Psychology, 1968, 4, 1-25.

(Received for publication May 20, 1976.) 